Methods Thirty Wistar albino rat pups were randomly divided into 3 groups: group 1, control; group 2, NEC and saline; group 3 , NEC and NAC treatment. NEC was induced by hyperosmolar enteral formula feeding and exposure to hypoxia after cold stress at $4^{\circ} \mathrm{C}$ and oxygen. The pups were killed on the fourth day and their intestinal tissues were harvested for biochemical and histopathologic analysis.

Results Mucosal injury scores and intestinal malondialdehyde levels in group 2 were found to be significantly higher than other groups $(p=<0.05)$. Intestinal superoxide dismutase activities in group 3 were significantly higher than group $2(p=0.018)$. Intestinal tissue TNF- $\alpha$ levels were significantly reduced with NAC treatment in group 3 compared to group $2(p<0.003)$.

Conclusions It is more likely that oxidative stress and inflammmatory mediators contributed to the pathogenesis of NEC and that NAC had a protective effect on intestinal injury through its antiinflammatory and antioxidant properties.

\section{ANALYSIS OF NEC INDUCING FACTORS OF PRETERM UNDER 1500G}

doi:10.1136/archdischild-2012-302724.0280

${ }^{1} \mathrm{JH}$ Jeon, ${ }^{2} \mathrm{SY}$ Bak. ${ }^{1}$ Neonatology; ${ }^{2}$ Pediatrics, CHA Kangnam Medical Center, CHA University, Seoul, Republic of Korea

Background and Aim NEC (Necrotizing Enterocolitis) is Various degrees of mucosal or transmural necrosis of the intestine and the most common life-threatening emergency of the gastrointestinal tract in the newborn period. Recently, Transfusion is reportred increased odds ratio of NEC after trasnfusion of red blood cells in premature infants.

We studied to investigate the relationship between the NEC inducing factors in preterm under $1500 \mathrm{~g}$ and red blood cell transfusion.

Method We analized Preterm infants $(\mathrm{n}=180)$ under $1500 \mathrm{~g}$ who were admitted at at Kangnam CHA Hospital NICU from January 2006 to december 2009. Preterm infants (G.A:24+6 wk 35+4wk, B.W $740 \mathrm{~g} \sim 1490 \mathrm{~g})$ were grouped NEC group (n=18, $\geq$ Stage $2 \mathrm{~b})$ and No-NEC group ( $n=162,<$ Stage $2 b$ ). NEC group was defined more stage $2 b$ by modified Bell's criteria. No-NEC group was defined under stage $2 b$. Statistics analysis used t-test, cross-tab, logistic regression by SPSS 12.0.

Result NEC group ( $\mathrm{n}=18,27.61 \pm 2.15 \mathrm{wk}, 1027.78 \pm 343.57 \mathrm{~g})$ was singnificant in Apgar $(1 \mathrm{~min})(3.33 \pm 1.5$ vs $4.39 \pm 1.6, \mathrm{p}=0.01)$, Apgar $(5 \mathrm{~min})(5.67 \pm 1.64$ vs $6.45 \pm 1.41, \mathrm{p}=0.03)$ score and $\mathrm{RDS}(100 \%$ vs $80.9 \%, p=0.04)$ with No-NEC group $(n=162,28.96 \pm 2.98$ wk, $1134.2 \pm 271.36 \mathrm{~g})$. Transfusion was not significant between NEC group (89\%) and No-NEC group $(77 \%)$. By multivariate logistic regression, gestation age and ventilator duration was correlation with NEC. The odds ratio of transfusion was 1.211 (95\%CI: $0.377 \sim 3.887, \mathrm{p}=0.748$ ) after adjustment of gestation age and Apgar score.

Abstract 280 Table 1 Clinical Characteristics of Preterm Infants

\begin{tabular}{llll}
\hline & NEC $(\mathbf{n}=\mathbf{1 8})$ & No-NEC (n-162) & P=value \\
\hline B.W(g) & $1027.78 \pm 343.57$ & $1134.2 \pm 271.36$ & NS \\
G.A(wk) & $27.61 \pm 2.15$ & $28.96 \pm 2.98$ & NS \\
Male gender(n) & $7(38.89 \%)$ & $77(47.53 \%)$ & NS \\
Apgar(1min) & $3.33 \pm 1.5$ & $4.39 \pm 1.64$ & 0.01 \\
Apgar(5min) & $5.67 \pm 1.64$ & $6.45 \pm 1.41$ & 0.03 \\
PDA(n) & $7(38.89 \%)$ & $47(29.01 \%)$ & NS \\
RDS(n) & $18(100.0 \%)$ & $131(80.86 \%)$ & 0.046 \\
ventilator(d) & $8.33 \pm 7.28$ & $6.99 \pm 11.22$ & NS \\
Trasfusion(n) & $16(88.89 \%)$ & $124(76.54 \%)$ & NS \\
\hline
\end{tabular}

Conclusion Apgar score and RDS was significant correlation with NEC. Transfusion was not significant correlation with NEC.

\section{PROTEIN C AND PROTEIN S IN THE DIAGNOSIS, MANAGEMENT AND PROGNOSIS OF PREMATURE INFANT WITH NECROTIZING ENTEROCOLITIS}

doi:10.1136/archdischild-2012-302724.0281

'LS Wang, ${ }^{2} \mathrm{GO}$ Cheng, ${ }^{2} \mathrm{Y}$ Cao, ${ }^{2} \mathrm{XM}$ Shao, ${ }^{2} \mathrm{WH}$ Zhou. 'Department of Neonatology; ${ }^{2}$ Children's Hospital, Fudan University, Shanghai, China

Objective In this prospective, observational study, we determined whether serum protein $\mathrm{C}$ and protein S (PC/PS) activity correlated with necrotizing enterocolitis (NEC) stages II and III in premature infant. We hypothesized that PC/PS measurement if used as an adjunct to abdominal radiographs would improve the identification and predication of infants with NEC.

Methods Serum PC/PS activity was measured according to protocol at early stage and recover stage of NEC. When clinical signs persisted and the initial abdominal radiographs were abnormal, follow-up radiographs were obtained. Results. Of 45 infants who were evaluated for gastrointestinal signs, only 2 had ileus or benign pneumatosis intestinalis with persistently normal PC/PS; gastrointestinal manifestations resolved within 48 hours, antibiotics were discontinued in $<48$ hours, and feedings were restarted early without complications. 35 infants had NEC stages II and III; all had decreased PC/PS activity regardless of their blood culture results. PC/PS activity negatively correlative with severity of NEC and positively correlative with the recover form NEC.

Conclusions In infants with suspected NEC, normal PC/PS values would favor aborted antibiotic therapy and early resumption of feedings. PC/PS becomes significantly lower in both stage II and stage III NEC. In infants with NEC, persistently decreased PC/PS after initiation of appropriate medical management suggests associated complications, which may require surgical intervention.

\section{MATERNAL PREECLAMPSIA IS ASSOCIATED WITH INCREASED RISK OF NECROTIZING ENTEROCOLITIS IN PRETERM INFANTS}

doi:10.1136/archdischild-2012-302724.0282

M Cetinkaya, H Ozkan, N Koksal. Uludağ University Medical Faculty, Bursa, Turkey

Objective The aim of this study was to evaluate the effect of maternal preeclampsia on development and severity of NEC in premature infants.

Methods This study consisted 2 groups of preterm infants ( $\leq 37$ gestational age): the study group contained preterm infants born to a preeclamptic mother and the comparison group contained preterm infants born to a normotensive mother. NEC was diagnosed according to clinical and radiographic findings, and it was classified according to modified Bell's criteria.

Result The study group consisted 174 premature infants born to preeclamptic mothers and the control group consisted 327 premature infants born to normotensive mothers. There were a total of 88 infants (40 infants in the study group and 48 infants in the control group) who had NEC diagnosis. The incidence of NEC in infants born to preeclamptic mothers $(22.9 \%)$ was significantly higher compared with those born to normotensive mothers (14.6\%). NEC was more advanced in preeclamptic mother infants. NEC developed significantly earlier in infants with NEC in the study group compared to those with NEC in the control group. The duration of NEC was also significantly longer in infants born to preeclamptic mothers.

Conclusion Maternal preeclampsia may be an important risk factor for development of NEC in premature infants as NEC incidence 